\title{
The Optical/Near-Infrared Light Curves of SN 2002ap for the First 1.5 Years after Discovery
}

\author{
Hiroyuki Tomita ${ }^{2}$, Jinsong Deng ${ }^{1,3}$, Keiichi Maeda ${ }^{4}$, Yuzuru Yoshii ${ }^{2,5}$, Ken'ichi Nomoto ${ }^{3,5}$, \\ Paolo A. Mazzali ${ }^{3,6,7}$, Tomoharu Suzuki ${ }^{3}$ Yukiyasu Kobayashi ${ }^{8}$, Takeo Minezaki ${ }^{2}$, Tsutomu \\ Aoki $^{2}$, Keigo Enya ${ }^{9}$, and Masahiro Suganuma ${ }^{8}$
}

\begin{abstract}
Late-time BVRIJHK photometry of the peculiar Type Ic SN 2002ap, taken between 2002 June 12 and 2003 August 29 with the MAGNUM telescope, is presented. The light curve decline rate is derived in each band and the color evolution is studied through comparison with nebular spectra and with SN 1998bw. Using the photometry, the OIR bolometric light curve is built, extending from before light maximum to day 580 after explosion. The light curve has a late-time shape strikingly similar to that of the hypernova SN 1998bw. The decline rate changes from 0.018 $\mathrm{mag}$ /day between day 130 and 230 to $0.014 \mathrm{mag} /$ day between day 270 and 580 . To reproduce the late-time light curve, a dense core must be added to the 1-D hypernova model that best fits the early-time observations, bringing the ejecta mass from $2.5 M_{\odot}$ to $3 M_{\odot}$ without much change in the kinetic energy, which is $4 \times 10^{51} \mathrm{ergs}$. This is similar to the case of other hypernovae and suggests asymmetry. A large $H$-band bump developed in the spectral energy distribution after $\sim$ day 300, probably caused by strong [Si I] $1.646 \mu \mathrm{m}$ and $1.608 \mu \mathrm{m}$ emissions. The near-infrared flux contribution increased simultaneously from $<30 \%$ to $>50 \%$ at day 580 . The near-infrared light curves were compared with those of other Type Ib/c supernovae, among which SN 1983I seems similar to SN 2002ap both in the near-infrared and in the optical.
\end{abstract}

Subject headings: supernovae: general-supernovae: individual (SN 2002ap)—supernovae: photometry

\footnotetext{
${ }^{1}$ Correspondence author's address: National Astronomical Observatories, Chinese Academy of Sciences, 20A Datun Road, Chaoyang District, Beijing 100012, China; jsdeng@bao.ac.cn

${ }^{2}$ Institute of Astronomy, School of Science, University of Tokyo, 2-21-1 Osawa, Mitaka, Tokyo 181-0015, Japan

${ }^{3}$ Department of Astronomy, School of Science, University of Tokyo, 7-3-1 Hongo, Bunkyo-ku, Tokyo 113-0033, Japan

${ }^{4}$ Department of Earth Science and Astronomy, Graduate School of Arts and Science, University of Tokyo, Meguro-ku, Tokyo 153-8902, Japan

${ }^{5}$ Research Center for the Early Universe, School of Science, University of Tokyo, 7-3-1 Hongo, Bunkyo-ku, Tokyo 113-0033, Japan

${ }^{6}$ Osservatorio Astronomico, Via Tiepolo 11, 34131 Trieste, Italy

${ }^{7}$ Max-Planck-Institut für Astrophysik, KarlSchwarzschild-Strasse 1, 85748 Garching, Germany

${ }^{8}$ National Astronomical Observatory, 2-21-1 Osawa, Mitaka, Tokyo 181-8588, Japan

${ }^{9}$ Institute of Space and Astronomical Science, Japan
}

\section{Introduction}

There is growing interest in Type Ib/c supernovae (SNe) following the discovery of a subclass that display very broad-lined spectra, indicating the existence of ejecta expanding with velocity $>0.1 c$. Models of the optical spectra and the light curves (LCs) of this high-velocity subclass, also called hypernovae (HNe, e.g., Nomoto et al. 2004), have concluded that these are the explosions of massive $\mathrm{C}+\mathrm{O}$ stars, producing up to 10 times the kinetic energy of normal core-collapse SNe (e.g., Iwamoto et al. 1998, 2000; Mazzali et al. 2003; Deng et al. 2005). It is unclear why some HNe are apparently associated with GRBs, i.e., SN 1998bw and GRB 980425, (Galama et al.

Aerospace Exploration Agency, 3-1-1, Yoshinodai, Sagamihara, Kanagawa 229-8510, Japan 
1998), SN 2003dh and GRB 030329 (Stanek et al. 2003), and SN 2003lw and GRB 031203 (Malesani et al. 2004), while others are not.

The Type Ic SN 2002ap, discovered in M74 (Nakano et al. 2002), is one of the nearest SNe in recent years. Its host galaxy has a distance modulus of only $29.5_{-0.2}^{+0.1}$ (Sharina, Karachentsev, \& Tikhonov 1996; Sohn \& Dvidge 1996; see also Hendry et al. 2005 for the latest review). TakadaHidai, Aoki, \& Zhao (2002) measured the total extinction toward the SN with a high-resolution spectrum and concluded that $E(B-V)=0.09 \pm$ 0.01 . We adopt these values in this paper.

Early-time optical spectroscopy and photometry was published by Mazzali et al. (2002), GalYam, Ofek, \& Shemmer (2002), Kinugasa et al. (2002), Foley et al. (2003), Pandey et al. (2003a), Vinkó et al. (2004), and others. Most authors emphasized the spectral similarity to the broad-lined SN 1998bw and SN 1997ef. The early-time light curve (LC), was much broader than that of the normal Type Ic SN 1994I, but was significantly fainter than that of SN 1998bw and peaked earlier.

Yoshii et al. (2003) (hereafter Paper I) presented early-time near-infrared (NIR) and optical photometry, obtained with the MAGNUM telescope. Using the $U B V R I J H K$ photometry, they built a well-sampled $O I R$ bolometric LC for SN 2002ap. NIR photometry has also been reported by Nishihara et al. (2002) and Mattila, Meikle, \& Chambers (2002). Gerardy et al. (2004) described the evolution of NIR spectra, which were dominated by lines of intermediate-mass elements (see also Danziger et al. 2002).

Mazzali et al. (2002) modelled the early-time optical spectra and bolometric LC using 1-D codes. They concluded that SN 2002ap was the energetic explosion of a $\mathrm{C}+\mathrm{O}$ star, having evolved from a star of 20-25 $M_{\odot}$ on the main sequence, and that it ejected $\sim 2.5 M_{\odot}$ material with a kinetic energy of $\sim 4 \times 10^{51} \mathrm{ergs}$, including $\sim 0.07$ $M_{\odot}{ }^{56} \mathrm{Ni}$. This places SN 2002ap at the low-mass low-energy end of hypernovae. They also constrained the explosion date to MJD $52300.0 \pm 0.5$ days. We use the explosion date as the reference point for any SN epoch in this paper. Based on observed limits in pre-discovery images, Smartt et al. (2002) argued that the progenitor was either a single W-R star of main-sequence mass $<40 M_{\odot}$ or more likely part of an interacting binary.

SN 2002ap was also monitored in the radio (Berger, Kulkarni, \& Chevalier 2002; Soderberg, Nakar, \& Kulkarni 2005), X-rays (Sutaria et al. 2003; Soria, Pian, \& Mazzali 2004), and UV (Soria \& Kong 2002). Berger, Kulkarni, \& Chevalier (2002) estimated quite a small energy in any relativistic material from the faintness of the radio. They addressed the material with velocities larger than the shock velocity $(\sim 0.3 c)$. Therefore, their results do not contradict the high energy obtained in the 1-D model for the bulk SN ejecta by Mazzali et al. (2002), whose ejecta velocity distribution has a cut-off at $70,000 \mathrm{~km} \mathrm{~s}^{-1}$. As a possible confirmation of this result, the shock velocity derived by Björnsson \& Fransson (2004) from models of the radio and X-ray observations was also $\sim 70,000$ $\mathrm{km} \mathrm{s}^{-1}$.

SN 2002ap was not apparently associated with a GRB (Hurley et al. 2002). However, Kawabata et al. (2002) discovered a polarized spectrum component that resembled the total flux spectrum but redshifted by $z \sim 0.3$ (see also Leonard et al. 2002; Wang et al. 2003). They proposed that this component is due to scattering from jet material. Totani (2003) further suggested that the jet may be too baryon-contaminated to trigger a GRB and that it was expanding almost freely and hence was radio quiet. Tominaga et al. (2004) reproduced a bump in the LC of the XRF 030723 afterglow with a SN 2002ap-like LC.

SN 2002ap was monitored into the late nebular phase. Foley et al. (2003) published optical photometry covering from 2002 June to December and spectroscopy continuing to 2003 February. The spectra show unusually strong and sharp-peaked $[\mathrm{O} \mathrm{I}]$ and $\mathrm{Mg} \mathrm{I}]$ emission lines (see also Leonard et al. 2002). Late-time spectroscopy was also obtained by other observers (Y. Qiu; K. Kawabata; S. B. Pandey; private communications), and late-time photometry by Vinkó et al. (2004) and Doroshenko et al. (2003).

It is also important to have intensive late-time NIR observations of Type Ib/c SNe. As demonstrated in the case of SN 1998bw (Patat et al. 2001; Sollerman et al. 2002), the NIR contribution to the total flux is quite large, in particular at late times. Late-time NIR observations may also reveal molecule and dust formation, if any exists (e.g., Gerardy et al. 2004). However, few Type 
$\mathrm{Ib} / \mathrm{c}$ SNe have been observed at late phases in the NIR. For SN 2002ap, the only late-time NIR observation published so far is a spectrum taken in 2002 August (Gerardy et al. 2004).

In this paper, we report on $B V R I J H K$ imaging photometry obtained for SN 2002ap between 2002 June 12 and 2003 August 29, completing the 1.5 years follow-up project with the MAGNUM telescope (see also Paper I). We describe the observations and data reduction and present our multicolor LCs in $\S 2$. An $O I R$ bolometric LC is then constructed using the photometry. In $\S 3$, we discuss the bolometric LC, analyze the evolution of the spectral energy distribution (SED), compare the late-time NIR LCs with those of other Type $\mathrm{Ib} / \mathrm{c}$ SNe, and present 1-D models for the bolometric LC.

\section{Imaging Photometry}

\subsection{Observations and Data Reduction}

Optical (Johnson-Cousins $B V R I$ ) and NIR $(J H K)$ imaging photometry of SN 2002ap $\left(\alpha_{2000}=\right.$ $\left.01^{h} 36^{m} 23 .{ }^{s} 85, \quad \delta_{2000}=+15^{\circ} 45^{\prime} 13 .^{\prime \prime} 2\right)$ was carried out using the MAGNUM $2 \mathrm{~m}$ telescope at the summit of Haleakala on the Island of Maui, Hawaii (Yoshii 2002). The multicolor imaging photometer mounted at the bent-Cassegrain focus has an SITe $1024 \times 1024$ pixel CCD with a scale of $0 .{ }^{\prime \prime} 277$ pixel $^{-1}$, for which the light is effectively received in a field of view of $430 \times 430$ pixel CCD or $119^{\prime \prime} \times 119^{\prime \prime}$, and also has an SBRC $256 \times 256$ pixel InSb with a scale of $0 .^{\prime \prime} 346$ pixel $^{-1}$ yielding a field of view of $88 .^{\prime \prime} 5 \times 88 .^{\prime \prime} 5$. A dichroic beam splitter enables simultaneous imaging through optical $(U B V R I)$ and NIR $\left(Z J H K^{\prime} K L^{\prime}\right)$ filters (Kobayashi et al. 1998a).

The observations were scheduled by the MAGNUM scheduler (Kobayashi et al. 1998b), except for the solar conjunction from March to June in 2003. Telescope dithering was performed with 6 or 10 -arcsec steps primary for $J H K$. The typical exposure time for one step was $190 \mathrm{sec}$ for $B, 95$ sec for $V, 65 \mathrm{sec}$ for each of $R I, 60 \mathrm{sec}$ for $J$ and $30 \mathrm{sec}$ for each of $H K$. As SN 2002ap faded, the number of dithering steps was increased from 6 to 12. The typical seeing size of the stellar image was 1.0-1.6 arcsec in $V$ and $0.85-1.4$ arcsec in $K$. Dome-flat images for $B V R I J H K$ and NIR dark images were obtained at the end of each night.
Image reduction was performed using our package of IRAF-based ${ }^{1}$ automated reduction software (MAGRED), which includes the standard corrections for bias and flat field frames. For more details see Paper I.

Two reference stars, $\mathrm{A}$ and $\mathrm{B}$, were observed in the same frame of SN 2002ap for differential photometry. One reference star $\left(\mathrm{A}, \alpha_{2000}=\right.$ $\left.01^{h} 36^{m} 19 . .^{s} 49, \delta_{2000}=+15^{\circ} 45^{\prime} 20 .^{\prime \prime} 8\right)$ was used for $B V R I$ as in Paper I. The other reference star $\left(\mathrm{B}, \alpha_{2000}=01^{h} 36^{m} 20 .{ }^{s} 32, \delta_{2000}=+15^{\circ} 44^{\prime} 54 .^{\prime \prime} 3\right.$ ) was used for $J H K$. In order to calibrate the magnitude of star B, NIR standard stars (Hunt et al. 1998) were also observed, whenever possible, either before or after the observation of SN 2002ap. The median magnitudes and the number of calibrations for star B in the InSb frame are $J(6)=15.69 \pm 0.02, H(6)=15.11 \pm 0.05$, and $K(6)=14.86 \pm 0.03$.

A variability check was made for stars A and $\mathrm{B}$ by increasing the total exposure time in proportion as SN 2002ap faded. Figure 1 shows the difference between their $B V R I$ magnitudes after 2003 July, when the optical flux of star B became detectable. (It was not possible to make the same plot in the NIR because star A was usually outside or on the edge of the InSb detector.) The error bars estimated from photon statistics are in good agreement with the dispersion of the data points, so that it is not necessary to regard stars $\mathrm{A}$ and $\mathrm{B}$ as variable.

Simple aperture photometry was used throughout, and the aperture magnitude of SN 2002ap was obtained as the magnitude difference between SN 2002ap and star A or B. An aperture size of 6.9 arcsec was adopted for all images, which minimizes the dispersion of the differential photometry. However, special care was taken for reliable magnitude determination of SN 2002ap, given the presence of a faint background object within a distance of about 1 arcsec. This object became distinguishable from SN 2002ap, when SN 2002ap became sufficiently faint after solar conjunction in 2003. In 2003 December, SN 2002ap faded compared to the background object, and it was easy

\footnotetext{
${ }^{1}$ IRAF is distributed by the National Optical Astronomy Observatories, which are operated by the Association of Universities for Research in Astronomy, Inc., under contract to the National Science Foundation.
} 
to resolve SN 2002ap and the object separately. Then, using the IRAF DAOPHOT package, the PSF magnitude of the object was measured several times when the seeing was relatively good. This way, the PSF magnitude of the object was determined as $B=21.7 \pm 0.5, V=21.6 \pm 0.2$, $R=21.25 \pm 0.12, I=20.62 \pm 0.17, J=20.0 \pm 0.1$, $H=19.3 \pm 0.1$, and $K=19.1 \pm 0.3$. These fluxes were subtracted from each aperture photometry of SN 2002ap.

Moreover, reference star B in NIR photometry has a faint nearby star which is about 4 mag fainter. Photometry was performed on the images where this faint nearby star was subtracted using IRAF DAOPHOT package.

\subsection{Observed Light Curves}

The $B V R I J H K$ data taken over 42 nights from June 12, 2002 to Aug 29, 2003 are tabulated in Table 1 . The uncertainty in each magnitude was derived combining in quadrature the magnitude dispersion from the frames obtained during the same night and the magnitude error of reference star A for $B V R I$, or B for $J H K$. A small error of 0.01 mag was added in quadrature for the $B V R I$ magnitudes, taking into account the nonlinearity correction error between SN 2002ap and reference star A. Another $0.01 \mathrm{mag}$ error caused by the seeing effect, arising from the profile difference between SN 2002ap and the reference star A, was also added. The absolute calibration error and the subtracted flux uncertainty of the background object were taken into account when evaluating the total photometry error. No color-term correction was applied to the magnitudes of SN 2002ap in the nebular phase, because strong line emissions and large color difference with respect to the main sequence star make this correction particularly difficult, as demonstrated by Menzies (1989).

We show the MAGNUM $U B V R I J H K$ LCs in Figure 2 (filled circles), combining the late-time data in Table 1 and the early-time data of Paper I. For comparison, we also show the $U B V R I$ photometry of Pandey et al. (2003a,b) (< 340 days; crosses), that of Foley et al. (2003) (<320 days; open circles), and the early-time $J H K$ photometry of Nishihara et al. (2002) ( $<50$ days; open circles). Obviously, our LCs have the largest time span. They are unique also in that both the early and late phase were well covered in NIR, the first time for any $\mathrm{SN} \mathrm{Ib/c.} \mathrm{To} \mathrm{guide} \mathrm{the} \mathrm{eye,} \mathrm{we} \mathrm{made}$ B-spline fits (solid lines) to the data, excluding the Pandey and Foley late-time ones.

It is clear from the figure that the decline of the late-time LC slowed down after September 2002 in several bands. We derived the decline rates and the date when the decline rate changed in each band by $\chi^{2}$-fitting the late-time MAGNUM data using a broken line (see Table 2). Before the change, which took place between about day 210 and 250, the decline rate was similar in all bands, i.e., about $0.016-0.022 \mathrm{mag} /$ day. The change is most profound in the NIR, as the $J$ and $H$ LCs flattened from $0.018 \mathrm{mag} /$ day to 0.012 and 0.008 $\mathrm{mag} /$ day, respectively. It is small for most optical bands, although the $V$ LC did flatten from 0.021 to $0.014 \mathrm{mag} /$ day. As a result, the NIR contribution to the total flux increased significantly going to very late times. This will be discussed further in the next section.

Most of our late-time data agree well with the Foley et al. (2003) ones, although their $B$ magnitudes are 0.1-0.2 mag brighter than ours after day 280 and their $I$ magnitudes 0.1-0.25 mag brighter after day 160. The Pandey et al. (2003b) latetime data are 0.1-0.15 mag brighter than ours in the $B$ band, 0.2-0.4 mag brighter in the $V$ band, and 0.1-0.2 mag fainter in the $I$ band. Those differences can be partly explained by measurement uncertainties inherent to practical broadband photometry of SNe (e.g., see Hamuy et al. 1990), since these objects have time-evolving and linedominated spectra of non-stellar nature. Our latetime $B V R I$ LCs can differ from the those of Vinkó et al. (2004) and Doroshenko et al. (2003) by as much as 0.3 mag. However, their data were less well sampled than ours.

The late-time evolution of the optical colors, $B-V, V-R$, and $R-I$, is very different between SN 2002ap and SN 1998bw, as shown in Figure 3. In particular, $V-R$ of SN 2002ap between day 140 and 330 is about $0.3-0.6$ mag larger and $B-V$ between day 200 and 340 about 0.3 mag smaller than in SN 1998bw. This can be easily understood within the context of spectroscopy. In the nebular phase, the spectra of both $\mathrm{SNe}$ were dominated by [O I] $\lambda \lambda 6300,6364$ in the $R$ band, [Fe II] multiplets in the $V$ and $B$ bands, and $\mathrm{Mg} \mathrm{I}] \lambda 4571$ in the $B$ band, while Ca II] $\lambda \lambda 7291,7324$ may also contribute to the $R$ and $I$ photometry and the 
Ca II NIR triplet to the $I$ photometry depending on the actual filter cut-off. As discussed by Foley et al. (2003), SN 2002ap has the strongest [O I] $\lambda \lambda 6300,6364$ and $\mathrm{Mg} \mathrm{I}] \lambda 4571$ nebular emissions of any SN published. However, its [Fe II] multiplets are much weaker than in SN 1998bw, a consequence of much less ${ }^{56} \mathrm{Ni}$ ejected by SN 2002ap. Between day 390 and $520, V-R$ and $R-I$ dropped and $B-V$ rose rapidly. This may indicate a flux shift from emissions of intermediate-mass elements to [Fe II] multiplets. Unfortunately, no observed spectra have so far been obtained for Type Ib/c $\mathrm{SNe}$ at such late times.

\section{Discussion}

\subsection{Bolometric Light Curve}

We built the $O I R$ bolometric LC integrating the $B V R I J H K$ broadband flux (see Paper I for details). A reddening of $E(B-V)=0.09 \pm 0.01$ was corrected for (Takada-Hidai, Aoki, \& Zhao 2002), and a distance modulus of 29.5 was adopted (but see Hendry et al. 2005).

The LC is shown in Figure 4, extending that in Paper I to $\sim 580$ days since explosion. It consists of three independent data sets, which agree with one another. Most of the data (filled circles), tabulated in Table 3 (late time) and Paper I (early time), were made from the MAGNUM photometry. We interpolated and extrapolated the observations to estimate the the $K$ magnitudes between day 280 and 340 and the $J$ and $I$ magnitudes at day 520. For epochs $<11$ days, the observations of Mattila, Meikle, \& Chambers (2002), Gal-Yam, Ofek, \& Shemmer (2002), and Cook et al. (2002) were used for interpolation. Bolometric magnitudes at day 407 and 578 (triangles) were converted from $H$ magnitudes using approximate bolometric corrections. The other two data sets were based on the Foley et al. (2003) (open circles) and Pandey et al. (2003a,b) (crosses) optical photometry, respectively, which was combined with the Nishihara et al. (2002) and our JHK photometry.

The bolometric LC at late time has a shape similar to that of SN 1998bw (Figure 4; squares), another hypernova, although the latter was much brighter. The SN 1998bw LC in Figure 4 is shifted down by $1.75 \mathrm{mag}$ to match the peak magnitude of SN 2002ap. Both LCs show a similar late-time slowing-down. For SN 2002ap, the decline rate changed from $\sim 0.018 \mathrm{mag} /$ day between day 130 and 230 to $\sim 0.014 \mathrm{mag} /$ day between day 270 and 580. For SN 1998bw, the decline rate was $\sim 0.019$ mag/day between day 70 and 220 and $\sim 0.014$ mag/day between day 320 and 540. For comparison, the late-time bolometric LC of another hypernova, SN $1997 \mathrm{ef}$, followed the decay rate of ${ }^{56} \mathrm{Co}$, i.e., $\sim 0.01 \mathrm{mag} /$ day (Mazzali et al. 2004). On the other hand, SN 2002ap reached its light maximum $\sim 5$ days earlier than SN 1998bw did, although their peak widths are actually comparable (Figure 4; inset).

\subsection{Spectral Energy Distribution}

We show the SED of SN 2002ap at 5 typical late epochs in Figure 5, where the monochromatic fluxes converted from the MAGNUM $B V R I J H K$ photometry are connected using spline-fitting curves. The observed upper limits of the $K$-band flux at day 393 and 520 are marked by arrows. Zero flux was assumed at both the the blue edge of the $B$ band and the red edge of the $K$ band. We used such SEDs to obtain the OIR bolometric magnitudes.

The SEDs are dominated by the flux in the $R$ and $I$ bands before about day 340-390, which was mainly due to strong [O I], Ca II], and Ca II emissions (Foley et al. 2003). The $B$ bump at the three intermediate epochs reflects the contribution of $\mathrm{Mg} \mathrm{I}] \lambda 4571$. After about day 340-390, the $V$ flux, attributed to [Fe II] multiplets, increased relative to that in other optical bands, while the $I$ band-dominated Ca II emissions died away.

The NIR flux contribution rose rapidly after $\sim$ day 300 (Figure 6). This can been seen in Figure 5 as a big $H$ bump in the SED and also in Figure 2 as a significant flattening of the $H$ and $J$ LCs. To explain this, we examined the synthesized NIR spectra of the models that reproduce the late-time optical spectra of SN 2002ap (P. A. Mazzali et al. 2005 , in preparation). The most likely candidates are the strong [Si I] $1.646 \mu \mathrm{m}$ and $1.608 \mu \mathrm{m}$ lines, while the $1.099 \mu \mathrm{m}$ line may account for the concurrent $J$-band flux increase. The lines were already developed in the day $\sim 200$ NIR spectrum of Gerardy et al. (2004), although they were still not as strong as a $1.5 \mu \mathrm{m}$ feature, possibly $\mathrm{Mg}$ I emission. We note in passing that the appearance of the CO first-overtone band in that spectrum 
suggested by those authors seems to coincide with the $K$-band LC steepening seen in Figure 2.

The late-time $H$-band spectra of SN $1983 \mathrm{~N}$, taken by Graham et al. (1986) and of very low resolution, showed a strong $1.65 \mu \mathrm{m}$ feature, which was explained by those authors as [Fe II] lines but was soon, together with an adjacent feature, reidentified as [Si I] lines by Oliva (1987). These [Si I] NIR features can also be found in the synthetic late-time spectra of Type Ib SNe computed by Fransson \& Chevalier (1989).

\subsection{Late-time NIR Light Curves of Type Ib/c Supernovae}

In Figure 7 we compare the late-time $J H K$ LCs of SN 2002ap with those of other Type Ib/c SNe (see Table 4). SN 1998bw and SN 1984L were 1.52.5 mag brighter than all other $\mathrm{SNe}$, indicating a large mass of ${ }^{56} \mathrm{Ni}$ ejected. The NIR LCs of SN 1984L were relatively slow, as was its late-time optical LC which was modelled using very massive ejecta but with normal kinetic energy (Swartz \& Wheeler 1991; Baron, Young, \& Branch 1993). The few points of SN 1998bw, when shifted down by $1.8 \mathrm{mag}(J), 1.5 \mathrm{mag}(H)$, and $1.7 \mathrm{mag}(K)$, respectively, fall on the LCs of SN 2002ap. The LCs of SN 1983N have later and broader peaks than those of SN 2002ap, but they become similar between in the $J$-band and $H$-band day $\sim 200$ and 350. On the other hand, the $J H K$ data of SN 1983I and 1982R seem to bridge the LC gap in SN 2002ap data between day 50 and day 140 (solar conjunction).

Figure 7 contradicts the picture that SNe 1983I, $1983 \mathrm{~N}$, and 1984L have similar JHK LCs (Elias et al. 1985). Type Ib/c SNe were first established as a new class by those authors, who showed that these SNe have similar $J H K$ LCs which are different from those of Type Ia $\mathrm{SNe}$ [and also by Wheeler \& Levreault (1985) through an analysis of the optical spectra]. They artificially shifted the data of SN 1983I and SN 1984L in both phase and brightness in order to match the NIR LCs of SN 1983N. Their SN 1983I data were assigned epochs 20 days later than ours, and SN 1984L 14 days earlier. Our epoch estimates are more reasonable because we based them on optical LCs and spectra which are better observed and understood than the NIR data.

\subsection{Was SN 1983I similar to SN 2002ap?}

Inspecting by eye the only published spectrum of SN 1983I (Wheeler et al. 1987), we noticed that it resembles those of SN 2002ap between 2002 February 16 and 22 (Kinugasa et al. 2002). The similarity can be seen not only in the overall shape and strongest line features, but also in relatively weak ones, like those near $4900 \AA, 5900 \AA$, and $6200 \AA$. The spectrum was taken on 1983 May 17,5 days after discovery. We estimate the epoch of that spectrum as $22 \pm 3$ days since explosion, through this comparison, and hence derive an explosion date of 1983 April 25.

The LCs of SN 1983I seem to match those of SN 2002ap not only in the NIR (Figure 7) but also in the optical (Figure 8), if our estimates of the explosion date and the distance modulus are correct. We adopt 31.0 as the distance modulus to the host galaxy of SN 1983I, NGC 4051 in the Ursa Major cluster (Tsvetkov 1985). This value is also close to the average of the distance modulus of the Ursa Major cluster (31.4, Tully \& Pierce 2000), and that corresponding to its radial velocity corrected for the Local Group infall onto Virgo (30.5, LEDA ${ }^{2}$; Paturel et al. 1997)

Therefore, SN 1983I could be a precedent of the unusual SN 2002ap, which lies at the lowmass low-energy end of hypernovae (Mazzali et al. 2002). However, its data are insufficient for us to make a solid conclusion.

\subsection{Modelling the Late-time Light Curve}

The late-time bolometric LC of SN 2002ap declines more slowly than that of the 1-dimensional (1-D) model of Mazzali et al. (2002) that best reproduced the early-time spectra and LC (see Figure 9 ; dotted line). This is expected, since the LC follows that of SN 1998bw closely in terms of the decline rate, as shown in Figure 4. The best-fitting 1-D model for the early-time spectra and LC of SN 1998bw also fails to explain its late-time spectrum and LC (Nakamura et al. 2001; Mazzali et al. 2001). To mimic the outcome of 2-dimensional jet-induced explosion calculations (Maeda et al. 2002), which applies to SN 1998bw and may also apply to SN 2002ap, Maeda et al. (2003) introduced a dense core. That core absorbs $\gamma$-rays ef-

\footnotetext{
${ }^{2} \mathrm{http://leda.univ-lyon1.fr/}$
} 
ficiently at late time. Using this structure, the slow LC decline of hypernovae was reproduced by those authors using a Monte-Carlo radiative transfer code but with simplified physics.

We tested the dense-core scenario on SN 2002ap using our sophisticated 1-D SN radiation hydrodynamical and $\gamma$-ray transfer code (Iwamoto et al. 2000; Mazzali et al. 2002; Deng et al. 2005). The best-fitting model (solid line) has $\sim 0.6 M_{\odot}$ ejecta below $3,000 \mathrm{~km} \mathrm{~s}^{-1}$, compared with $0.1 M_{\odot}$ in the model without a dense core, and $\sim 0.01 M_{\odot}$ low-velocity ${ }^{56} \mathrm{Ni}$. Compared with Mazzali et al. (2002), who modelled only the early-time observations, the total ejecta mass has increased from $2.5 M_{\odot}$ to $3 M_{\odot}$, but with little change in the total ${ }^{56} \mathrm{Ni}$ mass $\left(0.08 M_{\odot}\right)$ and the kinetic energy $\left(4 \times 10^{51} \mathrm{ergs}\right)$. A dense ejecta core is also required in 1-D late-time spectrum models (P. A. Mazzali et al. 2006, in preparation) in order to explain the observed sharp line cores of $[\mathrm{O} \mathrm{I}]$ and $\mathrm{Mg} \mathrm{I}]$ (Leonard et al. 2002; Foley et al. 2003).

A dense core cannot be formed in 1-D explosion simulations for hypernovae (Nakamura et al. 2001 ), but it is a natural product of 2 -D jetinduced explosions (Maeda et al. 2002). This strongly indicates asymmetry in the SN 2002ap explosion, an intrinsic feature also shared by other hypernovae. Evidence of asymmetry in SN 2002ap can also be found in spectropolarimetry (Kawabata et al. 2002; Leonard et al. 2002; Wang et al. 2003), which shows an intrinsic continuum polarization varying around $0.5 \%$, corresponding to an asphericity of $\sim 10 \%$ for the bulk of the ejecta.

We thank S. B. Pandey, E. Nishihara, and R. J. Foley for the electronic files of their photometric data. This work has been supported in part by the Grant-in-Aid for Scientific Research (16540229, 17030005 , \& 17033002 for KN) and the 21st Century COE program (QUEST) from the JSPS and MEXT in Japan, and by the National Science Foundation under Grant No. PHY99-07949.

\section{REFERENCES}

Baron, E., Young, T. R., \& Branch, D. 1993, ApJ, 409, 417
Berger, E., Kulkarni, S. R., \& Chevalier, R. A. 2002, ApJ, 577, L5

Björnsson, C.-I., \& Fransson, C. 2004, ApJ, 605, 823

Clocchiatti, A., Wheeler, J. C., Benett, S., \& Frueh, M. 1996, ApJ, 459, 547

Cook, L. M., Katkova, E. V., Sokolov, N. A., Guseva, I. S. 2002, IBVS, No. 5283

Danziger, J.,Della Valle, M., Palazzi, E., \& Pian, E. 2002, IAU Circ., No. 7838, 2

Deng, J., Tominaga, N., Mazzali, P., Maeda, K., \& Nomoto, K. 2005, ApJ, 624, 898

Doroshenko, V. T., Sergeev, S. G., Merkulova, N. I., \& Sergeeva, E. A. 2003, Astrophysics, 46, 365

Elias, J. H., Matthews, K., Neugebauer, G., \& Persson, S. E. 1985, ApJ, 296, 379

Foley, R. J., et al. 2003, PASP, 115, 1220

Fransson, C., \& Chevalier, R. A. 1989, ApJ, 343, 323

Galama, T. J., et al. 1998, Nature, 395, 670

Gal-Yam, A., Ofek, E. O., \& Shemmer, O. 2002, MNRAS, 332, L73

Gerardy, C. L., Fesen, R. A., Marion, G. H., Höflich, P., Wheeler, J. C., Nomoto, K., \& Motohara, K. 2004, in Cosmic Explosions in Three Dimensions, ed. P. Höflich, P. Kumer, \& J. C. Wheeler (Cambridge: Cambridge Univ. Press), 57

Graham, J. R., Meikle, W. P. S., Allen, D. A., Longmore, A. J., \& Williams, P. M. 1986, MNRAS, 218, 93

Hamuy, M., Suntzeff, N. B., Bravo, J., Phillips, M. M. 1990, AJ, 102, 888

Hendry, M. A., et al. 2005, MNRAS, 359, 906

Hunt, L. K., et al. 1998, AJ, 115, 2594

Hurley, K., et al. 2002, GCN. Circ. 1252 (http://gcn.gsfc.nasa.gov/gcn/gcn3/1252.gcn3)

Iwamoto, K., et al. 1998, Nature, 395, 672 
Iwamoto, K., et al. 2000, ApJ, 534, 660

Kawabata, K. S., et al. 2002, ApJ, 580, L39

Kinugasa, K., et al. 2002, ApJ, 577, L97

Kobayashi, Y., et al. 1998a, Proc. SPIE, 3352, 120

Kobayashi, Y., Yoshii, Y., Peterson, B. A., Minezaki, T., Enya, K., Suganuma, M., \& Yamamuro, T. 1998b, Proc. SPIE, 3354, 769

Leonard, D. C., Filippenko, A. V., Chornock, R. \& Foley, R. J. 2002, PASP, 114, 1333

Maeda, K., Nakamura, T., Nomoto, K., Mazzali, P. A., Patat, F., \& Hachisu, I. 2002, ApJ, 565, 405

Maeda, K., Mazzali, P. A., Deng, J., Nomoto, K., \& Yoshii, Y. 2003, ApJ, 593, 931

Malesani, D, et al. 2004, ApJ, 609, L5

Mattila, S., \& Meikle, W. P. S. 2001, MNRAS, 324,325

Mattila, S., Meikle, P., \& Chambers, K. 2002, IAU Circ., No. 7820, 1

Mazzali, P. A., Nomoto, K., Patat, F., \& Maeda, K. 2001, ApJ, 559, 1047

Mazzali, P. A., et al. 2002, ApJ, 572, L61

Mazzali, P. A., et al. 2003, ApJ, 599, L95

Mazzali, P. A., Deng, J, Maeda, K., Nomoto, K., Filippenko, A. V., \& Matheson, T. 2004, ApJ, 614, 858

Menzies, J. W. 1989, MNRAS, 237, 21

Nakamura, T., Mazzali, P. A., Nomoto, K., \& Iwamoto, K. 2001, ApJ, 550, 991

Nakano, S., Kushida, R., Kushida, Y., \& Li, W. 2002, IAU Circ., No. 7810, 1

Nishihara, E., Kinugasa, K., Hashimoto, O., Okuda, H., \& Yamaoka, H. 2002, in Proc. of the IAU 8th Asian-Pacific Regional Meeting, Vol. II, ed. S. Ikeuchi, J. Hearnshaw, \& T. Hanawa (Tokyo: ASJ), 347
Nomoto, K., Maeda, K., Mazzali, P. A., Umeda, H., Deng, J., \& Iwamoto, K. 2004, in "Stellar Collapse" (Astrophysics and Space Science) ed. C. L. Fryer (Dordrecht: Kluwer), 277 (astroph/0308136)

Oliva, E. 1987, ApJ, 321, L45

Pandey, S. B., Anupama, G. C., Sagar, R., Bhattacharya, D., Sahu, D. K., \& Pandey, J. C. 2003, MNRAS, 340, 375

Pandey, S. B., Sahu, D. K., Anupama, G. C., Bhattacharya, D., \& Sagar, R. 2003, Bull. Astr. Soc. India, 33, 351

Patat, F., et al. 2001, ApJ, 555, 900

Paturel, G., et al. 1997, A\&AS, 124, 109

Porter, A. C., \& Filippenko, A. V. 1987, AJ, 93, 1372

Schlegel, E. M., \& Kirshner, R. P. 1989, AJ, 98, 577

Schlegel, D. J., Finkbeiner, D. P., \& Davis, M. 1998, ApJ, 500, 525

Sharina, M. E., Karachentsev, I. D., \& Tikhonov, N. A. 1996, A\&AS, 119, 499

Sollerman, J, et al. 2002, A\&A, 386, 944

Smartt, S. J., Vreeswijk, P. M., Ramirez-Ruiz, E., Gilmore, G. F., Meikle, W. P. S., Ferguson, A. M., \& Knapen, J. H. 2002, ApJ, 572, L47

Soderberg, A., M., Nakar, E., \& Kulkarni, S. R. 2005, ApJ, submitted, preprint (astro$\mathrm{ph} / 0507147)$

Sohn, Y.-J., \& Dvidge, T. J. 1996, AJ, 111, 2280

Soria, R., \& Kong, A. K. H. 2002, ApJ, 572, L33

Soria, R., Pian, E., \& Mazzali, P. A. 2004, A\&A, 413, 107

Stanek, K. Z., et al. 2003, ApJ, 59, 17

Sutaria, F. K., Chandra, P., Bhatnagar, S., \& Ray, A. 2003, A\&A, 397, 1011

Swartz, D. A., \& Wheeler, J. C. 1991, ApJ, 379, L13 
Takada-Hidai, M., Aoki, W., \& Zhao, G. 2002, PASJ, 54, 899

Thim, F., Tammann, G. A., Saha, A., Dolphin, A., Sandage, A., Tolstoy, E., \& Labhardt, L. 2003, ApJ, 590, 256

Tominaga, N., Deng, J., Mazzali, P. A., Maeda, K., Nomoto, K., Pian, E., Hjorth, J., \& Fynbo, J. P. U. 2004, ApJ, 612, L105

Totani, T. 2003, ApJ, 598, 1151

Tsvetkov, D. Y. 1985, Sov. Astron., 29, 211

Tsvetkov, D. Y. 1987, Sov. Astron. Lett., 13, 376

Tully, R. B., \& Pierce, M. J., 2000, ApJ, 533, 744

Vinkó, J., et al. 2004, A\&A, 427, 453

Wang, L., Baade, D., Höflich, P., \& Wheeler, J. C. 2003, ApJ, 592, 457

Wheeler, J. C., \& Levreault, R. 1985, ApJ, 294, L17

Wheeler, J. C., Harkness, R. P., Barker, E. S., Cochran, A. L., \& Wills, D. 1987, ApJ, 313, L69

Yoshii, Y. 2002, in New Trends in Theoretical and Observational Cosmology, ed. K. Sato \& T. Shiromizu (Tokyo: Univ. Acad. Press), 235

Yoshii, Y., et al. 2003, ApJ, 592,467 (Paper I)

This 2-column preprint was prepared with the AAS LATEX macros v5.2.
Fig. 1. - Difference between observed magnitudes of two reference stars $[\Delta m \equiv m(\mathrm{~A})-m(\mathrm{~B})]$ in the $B V R I$ bands for MAGNUM observations after 2003 July. The mean difference $\langle\Delta m\rangle$ is shifted to zero. Error bars are estimated from photon statistics. The standard deviation of data distribution and mean error from photon statistics are $0.042 / 0.053(B), 0.032 / 0.030(V), 0.020 / 0.020$ $(R)$, and $0.014 / 0.010(I)$. The computed reduced chi-square values were 0.67 for $B, 1.11$ for $V, 1.23$ for $R$ and 1.33 for $I$.

Fig. 2.- $U B V R I J H K$ LCs of SN 2002ap for the first 1.5 years after discovery obtained with the MAGNUM telescope (filled circles). Also shown are the $U B V R I$ data reported by Foley et al. (2003) (open circles) and Pandey et al. (2003a,b) (crosses) and the early-time $J H K$ data by Nishihara et al. (2002) (also open circles). The LCs are shifted vertically in order to avoid overlap. Solid lines are B-spline fittings to the data, excluding the Pandey and Foley late-time ones.

Fig. 3. - Evolution of optical colors, $B-V, V-R$, and $R-I$, of SN 2002ap compared with that of SN 1998bw. The colors of SN 2002ap are calculated from the MAGNUM photometry, corrected for $E(B-V)=0.09$, while those of SN 1998bw from the photometry in Galama et al. (1998), Patat et al. (2001), and Sollerman et al. (2002), corrected for $A(V)=0.1$.

Fig. 4.- $O I R$ bolometric LC of SN 2002ap constructed using the MAGNUM $U B V R I J H K$ photometry, assuming $m-M=29.5$ and $E(B-V)=$ $0.09 \pm 0.01$, compared with that of SN 1998bw $[z=0.0085, m-M=32.76$, and $A(V)=0.1 \pm 0.1]$. Also shown are bolometric magnitudes based on the Foley et al. (2003) and Pandey et al. (2003a,b) optical photometry, which are combined with the MAGNUM and Nishihara et al. (2002) JHK photometry, and tho ones estimated from the MAGNUM $H$ photometry using approximate bolometric corrections. The SN 1998bw bolometric LC is shifted down by $1.75 \mathrm{mag}$ to match the peak brightness of SN 2002ap. It is constructed using the photometry in Galama et al. (1998), Patat et al. (2001), and Sollerman et al. (2002).

Fig. 5.- SEDs of SN 2002ap at 5 typical late epochs, constructed using the MAGNUM photom- 
etry. The BVRIJHK monochromatic flux is connected using spline-fitting curves. Capped arrows represent flux upper limits in the $K$ band.

Fig. 6.- Contribution of NIR flux to the total $O I R$ bolometric flux in SN 2002ap compared with that in SN 1998bw.

Fig. 7.- Absolute $J$ (top), $H$ (middle), and $K$ (bottome) LCs of the Type Ib/c SNe whose latetime NIR photometry are available. See Table 4 for data references and adopted SN parameters.

Fig. 8. - Absolute $B$ and $V$ LCs of the Type Ic SN 1983I (Tsvetkov 1985), assuming $m-M \approx 31.0$ and an explosion date of 1983 April 25 (see Table 4), compared with those of SN 2002ap.

Fig. 9.- $O I R$ bolometric LC of SN 2002ap compared with the best-fitting LCs of the 1-D ejecta model with a dense core at $v<3,000 \mathrm{~km} \mathrm{~s}^{-1}$ (total $M_{\mathrm{ej}} \approx 3 M_{\odot}$ ), and that without a dense core (total $M_{\mathrm{ej}} \approx 2.5 M_{\odot}$ ). Both models have total kinetic energy of $4 \times 10^{51} \mathrm{ergs}$ and $0.08 \mathrm{M}_{\odot}{ }^{56} \mathrm{Ni}$. 







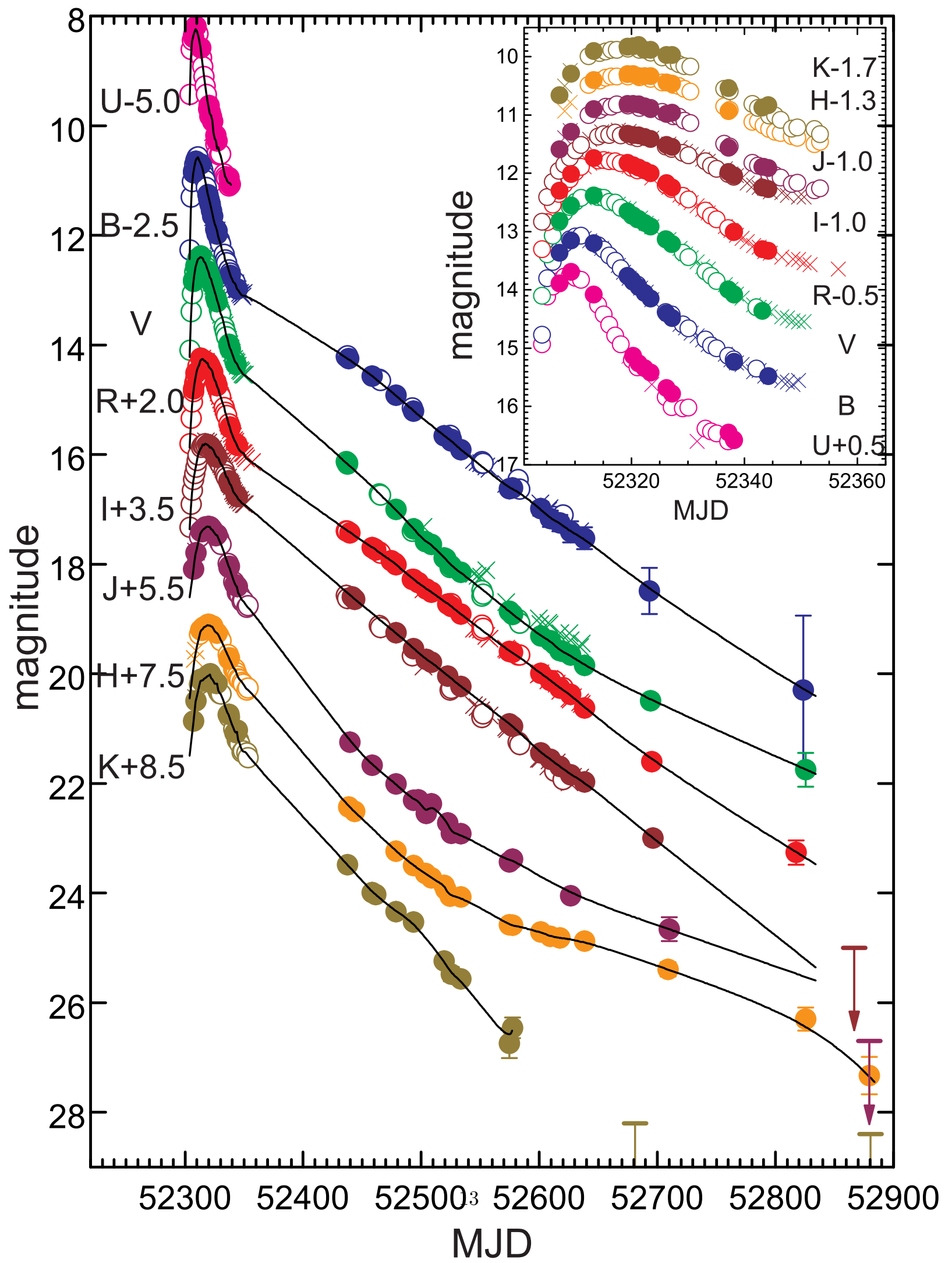




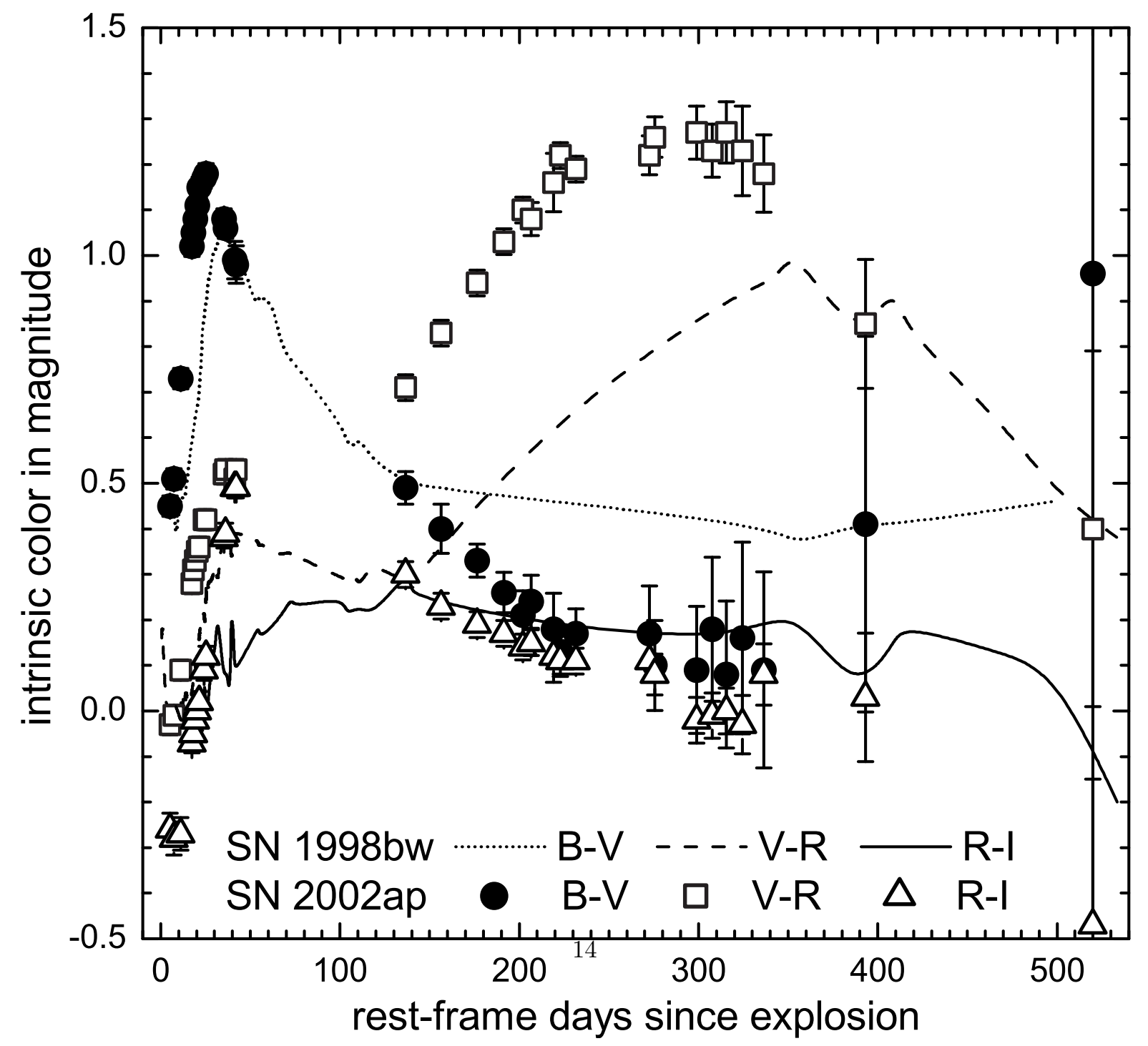




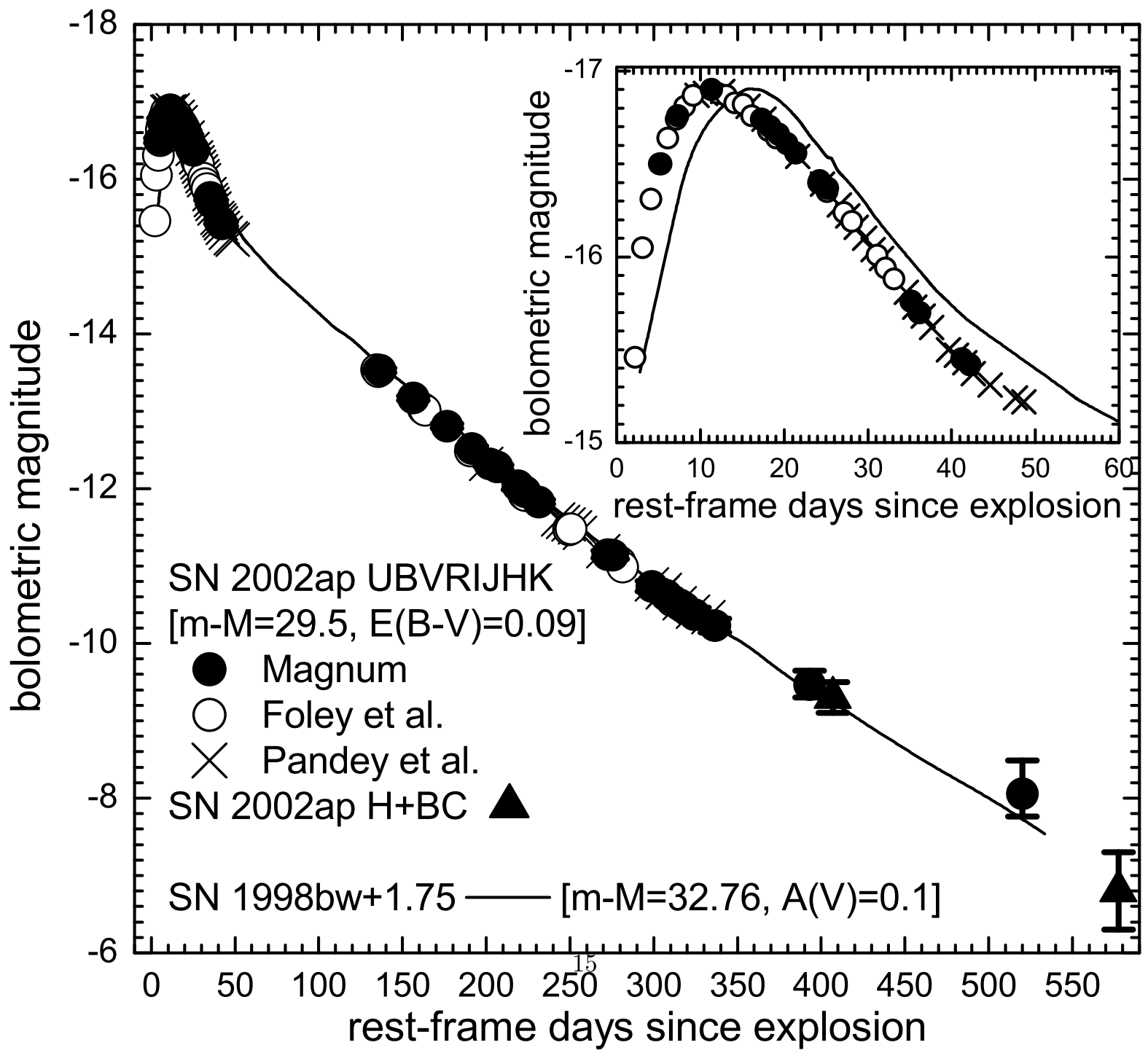









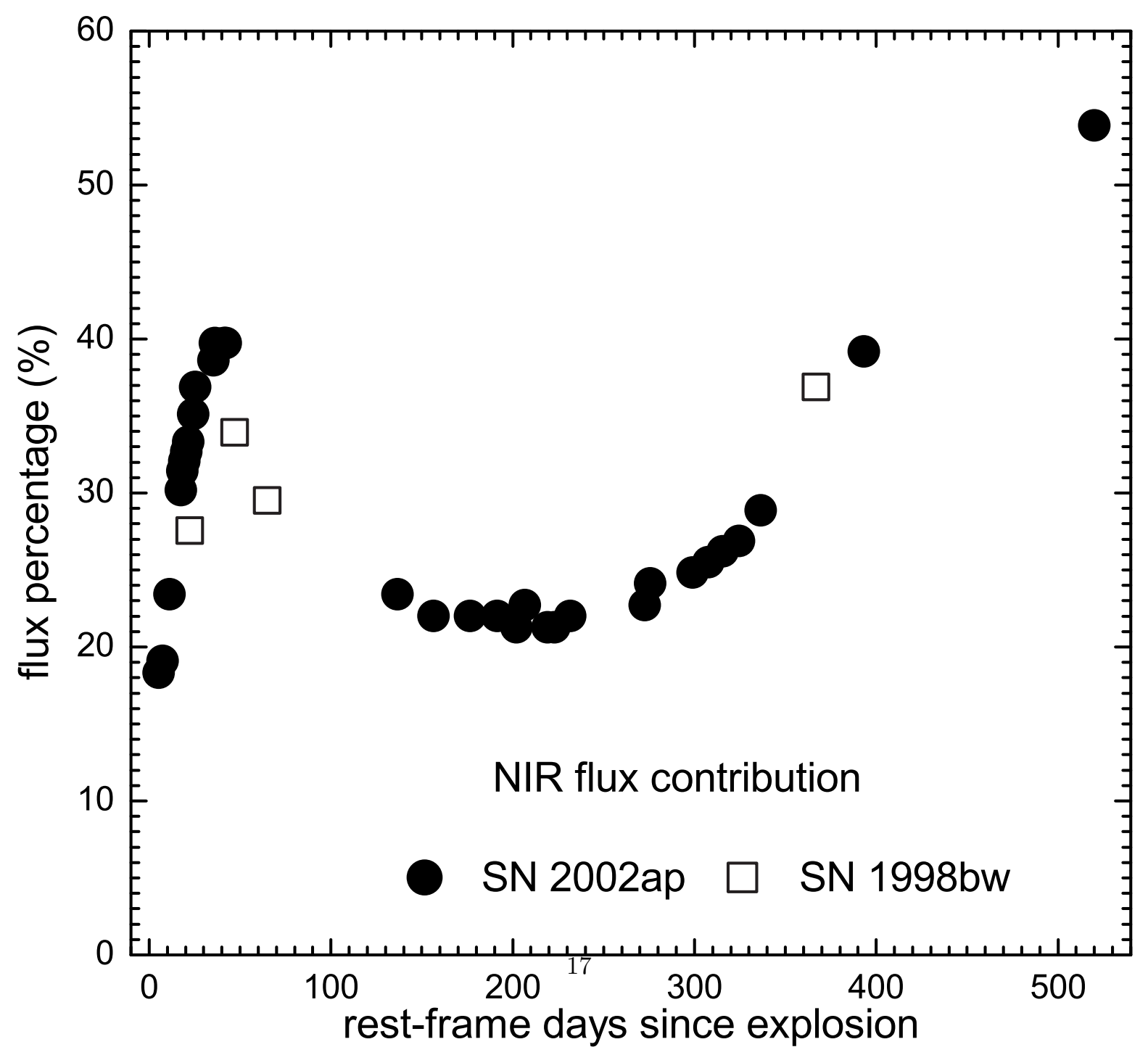




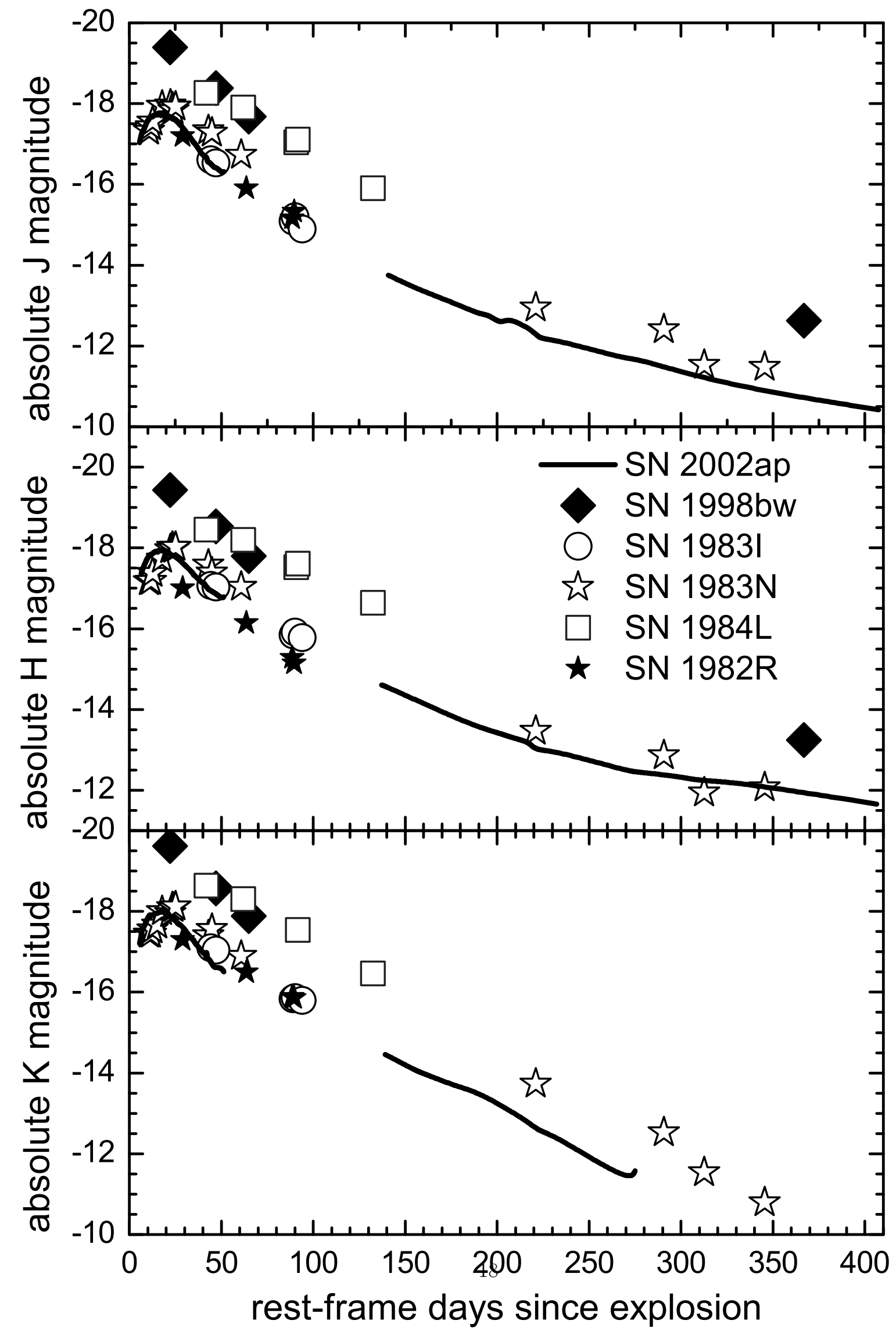




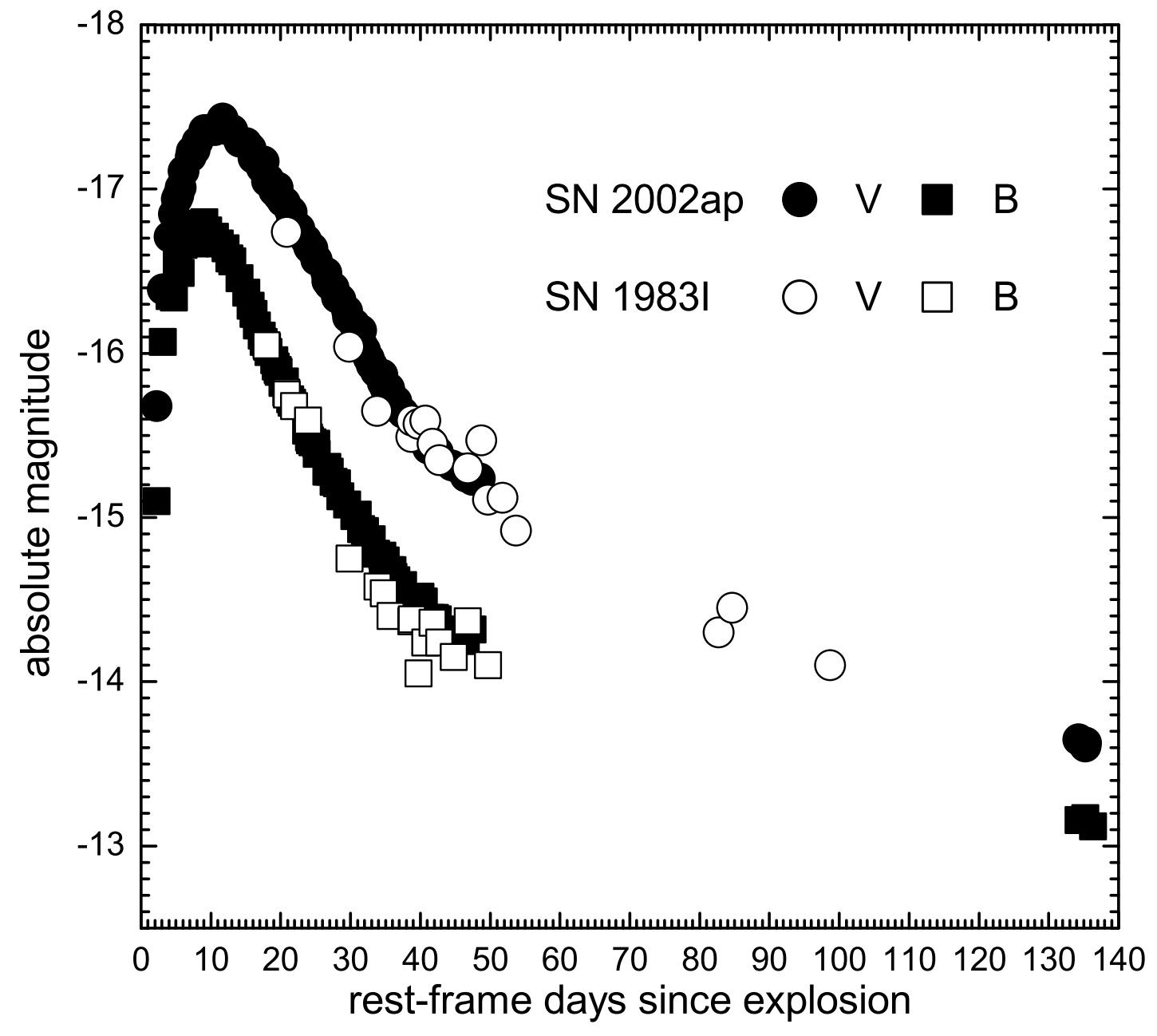




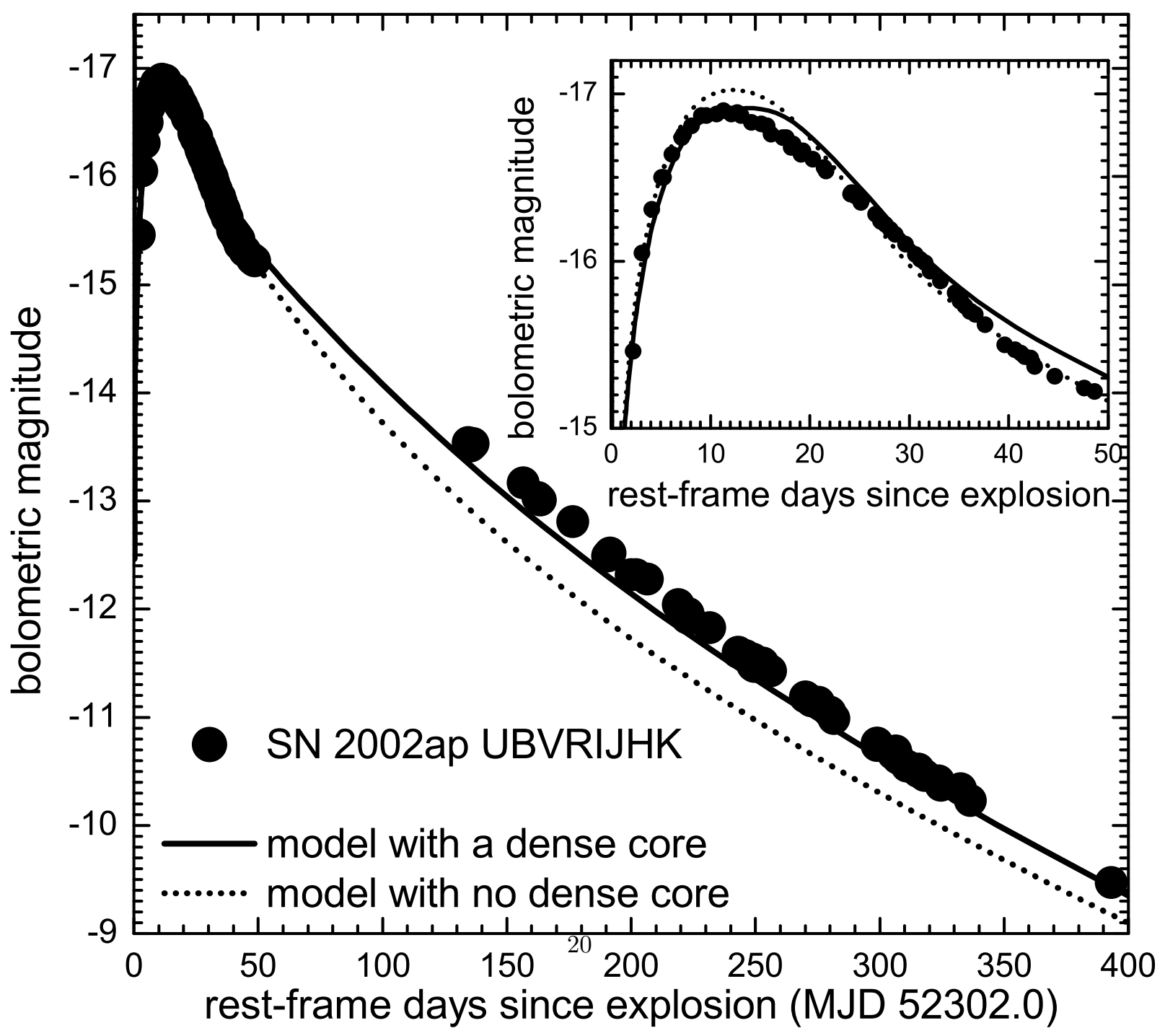


TABLE 1

DATA OF LIGHT CURVES OF SN 2002AP

\begin{tabular}{|c|c|c|c|c|c|c|c|c|c|}
\hline UT & MJD & $m_{B}$ & $m_{V}$ & $m_{R}$ & $m_{I}$ & $m_{J}$ & $m_{H}$ & $m_{K}$ & Weather \\
\hline Jun 12.61 & 52437.61 & & $16.15 \pm 0.02$ & $\ldots$ & $\ldots$ & $\ldots$ & . & $14.98 \pm 0.04$ & clear \\
\hline Jun 13.60 & 438.60 & $16.75 \pm 0.03$ & $\ldots$ & 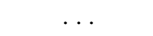 & $\ldots$ & & $14.93 \pm 0.05$ & $\ldots$ & clear \\
\hline Jun 14.60 & 2439.60 & $\ldots$ & $\ldots$ & $15.43 \pm 0.02$ & $\ldots$ & $15.75 \pm 0.03$ & $\ldots$ & $\ldots$ & clear \\
\hline Jun 16.60 & 2441.60 & $\ldots$ & $\ldots$ & $\ldots$ & $15.10 \pm 0.02$ & $\ldots$ & & $\ldots$ & clear \\
\hline Jun 18.60 & 52443.60 & $\ldots$ & $\ldots$ & $\ldots$ & $15.14 \pm 0.02$ & & $15.01 \pm 0.05$ & $\ldots$ & clear \\
\hline Jul 3.56 & 52458.56 & $17.07 \pm 0.05$ & $\ldots$ & $15.70 \pm 0.02$ & $\ldots$ & $16.17 \pm 0.04$ & $\ldots$ & $15.49 \pm 0.06$ & thin cloud \\
\hline Jul 5.60 & 52460.60 & $\ldots$ & $\ldots$ & $15.72 \pm 0.02$ & $\ldots$ & $\ldots$ & $\ldots$ & $\ldots$ & thin cloud \\
\hline Jul 6.55 & 2461.55 & $\ldots$ & $\ldots$ & $15.74 \pm 0.02$ & $\ldots$ & $\ldots$ & $\ldots$ & $15.53 \pm 0.08$ & cloudy \\
\hline Jul 19.56 & 474.56 & $\ldots$ & $\ldots$ & $15.94 \pm 0.02$ & $\ldots$ & $\ldots$ & $\ldots$ & $\ldots$ & clear \\
\hline Jul 22.56 & 2477.56 & $\ldots$ & $\ldots$ & $15.98 \pm 0.02$ & $\ldots$ & $\ldots$ & $\ldots$ & $\ldots$ & clear \\
\hline Jul 23.51 & 52478.51 & $17.42 \pm 0.03$ & $17.00 \pm 0.02$ & $16.01 \pm 0.02$ & $15.75 \pm 0.02$ & $16.51 \pm 0.03$ & $15.74 \pm 0.06$ & $15.84 \pm 0.06$ & clear \\
\hline Aug 7.47 & 52493.47 & $17.71 \pm 0.04$ & $17.36 \pm 0.02$ & $16.28 \pm 0.02$ & $16.04 \pm 0.02$ & $16.81 \pm 0.04$ & $16.00 \pm 0.07$ & $16.03 \pm 0.09$ & thin cloud \\
\hline Aug 11.59 & 52497.59 & $\ldots$ & & $16.34 \pm 0.02$ & $\ldots$ & $16.80 \pm 0.04$ & & $\ldots$ & thin cloud \\
\hline Aug 17.61 & 52503.61 & $\ldots$ & $17.59 \pm 0.02$ & & & & $16.14 \pm 0.06$ & $\ldots$ & thin cloud \\
\hline Aug 18.44 & 52504.44 & $\ldots$ & & $16.45 \pm 0.02$ & $16.24 \pm 0.02$ & $17.05 \pm 0.04$ & & $\ldots$ & clear \\
\hline Aug 22.54 & 52508.54 & $\ldots$ & $17.64 \pm 0.03$ & $16.51 \pm 0.02$ & $16.29 \pm 0.02$ & $16.87 \pm 0.04$ & $16.22 \pm 0.07$ & $\ldots$ & cloudy \\
\hline Sep 2.44 & 52519.44 & $18.16 \pm 0.05$ & $17.89 \pm 0.02$ & 2010 & $\ldots$ & 年 & $16.36 \pm 0.07$ & $16.74 \pm 0.08$ & clear \\
\hline Sep 3.61 & 52520.61 & $\ldots$ & $17.90 \pm 0.02$ & $\ldots$ & $\ldots$ & $\ldots$ & $16.43 \pm 0.07$ & $\ldots$ & thin cloud \\
\hline Sep 5.59 & 52522.59 & $\ldots$ & & $16.73 \pm 0.02$ & $16.54 \pm 0.02$ & $17.22 \pm 0.04$ & & $\ldots$ & thin cloud \\
\hline Sep 7.61 & 52524.61 & $\ldots$ & $18.02 \pm 0.02$ & $\ldots$ & $\ldots$ & & $16.56 \pm 0.07$ & $\ldots$ & cloudy \\
\hline Sep 8.41 & 52525.41 & $18.25 \pm 0.05$ & & $16.77 \pm 0.02$ & $\ldots$ & $17.41 \pm 0.07$ & & $16.98 \pm 0.13$ & cloudy \\
\hline Sep 16.54 & 52533.54 & $18.41 \pm 0.05$ & $18.15 \pm 0.02$ & $16.91 \pm 0.02$ & $16.73 \pm 0.02$ & $17.42 \pm 0.04$ & $16.57 \pm 0.06$ & $17.07 \pm 0.10$ & clear \\
\hline Oct 27.52 & 52574.52 & $19.12 \pm 0.10$ & $18.86 \pm 0.03$ & $17.59 \pm 0.03$ & $17.41 \pm 0.03$ & $17.93 \pm 0.07$ & $17.08 \pm 0.08$ & $18.24 \pm 0.27$ & clear \\
\hline Oct 30.45 & 52577.45 & $19.11 \pm 0$ & $18.92 \pm$ & $17.61 \pm 0$ & $17.46 \pm 0$ & $17.88 \pm 0.06$ & $17.09 \pm 0$ & $17.96 \pm 0.19$ & thin cloud \\
\hline Nov 23.22 & 52601.22 & $19.49 \pm 0.13$ & $19.31 \pm 0.05$ & $17.99 \pm 0.03$ & $17.94 \pm 0.04$ & $\ldots$ & $17.21 \pm 0.07$ & $\cdots$ & clear \\
\hline Dec 1.39 & 52609.39 & $19.67 \pm 0.15$ & $19.40 \pm 0$ & $18.12 \pm 0.03$ & $18.06 \pm 0.04$ & $\ldots$ & $17.29 \pm 0.07$ & $\ldots$ & clear \\
\hline Dec 9. & 5 & $19.75 \pm$ & 19 & & & & $17.32 \pm 0.07$ & $\ldots$ & clear \\
\hline Dec 18.37 & 52626.37 & $19.91 \pm 0$ & $19.66 \pm 0$ & $18.38 \pm 0.04$ & $18.34 \pm 0.05$ & $18.55 \pm 0.08$ & & $\ldots$ & clear \\
\hline Dec 30.37 & 52638.37 & $20.03 \pm 0.20$ & $19.85 \pm 0$ & $18.62 \pm 0.03$ & $18.47 \pm 0.06$ & $\ldots$ & $17.38 \pm 0.08$ & $\ldots$ & clear \\
\hline Feb 1 & 5268 & & $\ldots$ & $\ldots$ & $\ldots$ & $\ldots$ & $\ldots$ & $<19.7$ & clear \\
\hline Feb 23.22 & 52693.22 & $20.99 \pm 0.42$ & $\ldots$ & $\ldots$ & $\ldots$ & $\ldots$ & & $\ldots$ & clear \\
\hline Feb 24.23 & 52694 . & $\ldots$ & $20.49 \pm 0.11$ & & $\ldots$ & $\ldots$ & $\ldots$ & $\ldots$ & clear \\
\hline Feb 25.23 & 52695.23 & $\ldots$ & $\ldots$ & $19.60 \pm 0.06$ & $\ldots$ & $\ldots$ & $\ldots$ & $\ldots$ & clear \\
\hline Feb 26.23 & 52696.23 & $\ldots$ & $\ldots$ & $\ldots$ & $19.50 \pm 0.12$ & $\ldots$ & .. & $\ldots$ & clear \\
\hline Mar 11.21 & 52709.21 & $\ldots$ & $\ldots$ & $\ldots$ & $\ldots$ & $\ldots$ & $17.89 \pm 0.13$ & $\ldots$ & clear \\
\hline Mar 12.22 & 52710.22 & $\ldots$ & $\ldots$ & $\ldots$ & $\ldots$ & $19.16 \pm 0.22$ & $\ldots$ & $\ldots$ & clear \\
\hline Jun 27.62 & 52817.62 & $\ldots$ & $\ldots$ & $21.26 \pm 0.22$ & $\ldots$ & $\ldots$ & $\ldots$ & $\ldots$ & clear \\
\hline Jul 3.60 & 52823.60 & $22.80 \pm 1.36$ & $\ldots$ & $\ldots$ & $\ldots$ & $\ldots$ & $\ldots$ & $\ldots$ & clear \\
\hline Jul 5.60 & 52825.60 & $\ldots$ & $21.75 \pm 0.31$ & $\ldots$ & $\ldots$ & $\ldots$ & $18.80 \pm 0.21$ & $\ldots$ & clear \\
\hline Aug 15.49 & 52866.49 & $\ldots$ & $\ldots$ & $\ldots$ & $<21.5$ & $\ldots$ & $\cdots$ & $\ldots$ & thin cloud \\
\hline Aug 28.50 & 52879.50 & $\ldots$ & $\ldots$ & $\ldots$ & $\ldots$ & $<21.2$ & $19.83 \pm 0.34$ & $\ldots$ & clear \\
\hline Aug 29.50 & 52880.50 & $\ldots$ & $\ldots$ & $\ldots$ & $\ldots$ & & & $<19.9$ & clear \\
\hline
\end{tabular}


TABle 2

DeCline Rates of Light CURVES of SN 2002AP IN The Nebular Phase

\begin{tabular}{|c|c|c|c|c|c|c|c|c|}
\hline & $B$ band & $V$ band & $R$ band & $I$ band & $J$ band & $H$ band & $K$ band & Bolometric \\
\hline $\begin{array}{l}\text { Epoch range (MJD) } \\
\text { Decline rate (mag/day) }\end{array}$ & $\begin{array}{c}52438.6-52533.5 \\
0.017 \pm 0.0004\end{array}$ & $\begin{array}{c}52437.6-52533.5 \\
0.021 \pm 0.0002\end{array}$ & $\begin{array}{c}52439.6-52817.6 \\
0.016 \pm 0.0001\end{array}$ & $\begin{array}{c}52441.6-52696.2 \\
0.017 \pm 0.0001\end{array}$ & $\begin{array}{l}52439.6-52508.5 \\
0.018 \pm 0.001\end{array}$ & $\begin{array}{c}52438.6-52524.6 \\
0.018 \pm 0.001\end{array}$ & $\begin{array}{c}52437.6-52577.5 \\
0.022 \pm 0.001\end{array}$ & $\begin{array}{c}52438.5-52533.5 \\
0.018 \pm 0.0003\end{array}$ \\
\hline $\begin{array}{l}\text { Epoch range (MJD) } \\
\text { Decline rate (mag/day) }\end{array}$ & $\begin{array}{c}52574.5-52823.6 \\
0.015 \pm 0.002\end{array}$ & $\begin{array}{c}52574.5-52825.6 \\
0.014 \pm 0.001\end{array}$ & $\begin{array}{l}\cdots \\
\cdots\end{array}$ & $\begin{array}{l}\cdots \\
\cdots\end{array}$ & $\begin{array}{l}52522.6-52710.2 \\
0.012 \pm 0.001\end{array}$ & $\begin{array}{c}52533.5-52879.5 \\
0.008 \pm 0.0005\end{array}$ & $\begin{array}{l}\cdots \\
\cdots\end{array}$ & $\begin{array}{c}52574.5-52879.5 \\
0.014 \pm 0.001\end{array}$ \\
\hline Date of change (MJD) & $52545 \pm 5$ & $52535 \pm 1$ & & $\ldots$ & $52514 \pm 4$ & $52532 \pm 4$ & $\ldots$ & $52558 \pm 3$ \\
\hline
\end{tabular}


TABLE 3

BOLOMETRIC MAGNITUDES OF SN 2002AP

\begin{tabular}{cccc}
\hline \hline MJD a & Day & $M_{\text {bol }}$ & $L_{\text {NIR }} / L_{\text {bol }}$ \\
\hline 52438.5 & 136.5 & $-13.53 \pm 0.03$ & $23 \%$ \\
52458.5 & 156.5 & $-13.17 \pm 0.03$ & $22 \%$ \\
52478.5 & 176.5 & $-12.81 \pm 0.03$ & $22 \%$ \\
52493.5 & 191.5 & $-12.52 \pm 0.03$ & $22 \%$ \\
52504.0 & 202.0 & $-12.32 \pm 0.03$ & $21 \%$ \\
52508.5 & 206.5 & $-12.28 \pm 0.03$ & $23 \%$ \\
52521.0 & 219.0 & $-12.04 \pm 0.05$ & $21 \%$ \\
52525.0 & 223.0 & $-11.96 \pm 0.03$ & $21 \%$ \\
52533.5 & 231.5 & $-11.83 \pm 0.03$ & $22 \%$ \\
52574.5 & 272.5 & $-11.15 \pm 0.05$ & $23 \%$ \\
52577.5 & 275.5 & $-11.14 \pm 0.05$ & $24 \%$ \\
52601.0 & 299.0 & $-10.74 \pm 0.07$ & $25 \%$ \\
52609.5 & 307.5 & $-10.62 \pm 0.07$ & $26 \%$ \\
52617.5 & 315.5 & $-10.50 \pm 0.07$ & $26 \%$ \\
52626.5 & 324.5 & $-10.37 \pm 0.09$ & $29 \%$ \\
52638.5 & 336.5 & $-10.23 \pm 0.09$ & $29 \%$ \\
52695.0 & 393.0 & $-9.47_{-0.18}^{+0.17}$ & $39 \%$ \\
52709.0 & 407.0 & $-9.3 \pm 0.2$ & $\ldots$ \\
52822.0 & 520.0 & $-8.06_{-0.43}^{+0.30}$ & $54 \%$ \\
52879.5 & 577.5 & $-6.8 \pm 0.5$ & $\ldots$ \\
\hline
\end{tabular}

${ }^{a}$ With an accuracy of 0.5 days due to data interpolation/extrapolation.

${ }^{\mathrm{b}} \mathrm{With}$ respect to the explosion date (MJD 52302.0) 
TABLE 4

TyPe Ib/C SUPERNOVAE With LATE-TIME NIR PHOTOMETRY

\begin{tabular}{|c|c|c|c|c|c|}
\hline & SN 1982R & SN 1983I & SN $1983 N$ & SN 1984L & SN 1998bw \\
\hline Spectroscopic type & $\mathrm{Ib} / \mathrm{c}$ & Ic & $\mathrm{Ib}$ & $\mathrm{Ib}$ & Ic, hypernova \\
\hline Distance modulus $^{\text {a }}$ & 31.2 & 31.0 & 28.25 & 31.5 & 32.76 \\
\hline Extinction $A(V)^{\mathrm{b}}$ & 0.5 & 0.04 & 0.2 & 0.09 & $0.1 \pm 0.1$ \\
\hline Explosion date (UT) ${ }^{\mathrm{c}}$ & 1982 September 28 & 1983 April 25 & 1983 July 1 & 1984 August 5 & 1998 April 25.9 \\
\hline $\begin{array}{l}\text { JHK photometric } \\
\text { data sources }\end{array}$ & Mattila \& Meikle (2001) & Elias et al. (1985) & $\begin{array}{c}\text { Elias et al. (1985), } \\
\text { Mattila \& Meikle (2001) }\end{array}$ & Elias et al. (1985) & $\begin{array}{l}\text { Patat et al. (2001), } \\
\text { Sollerman et al. (2002) }\end{array}$ \\
\hline
\end{tabular}

${ }^{\mathrm{a}} h_{0}=0.72, \Omega_{\Lambda}=0.7, \Omega_{M}=0.3 ; 1982 \mathrm{R} \& 1984 \mathrm{~L}$, radial velocity corrected for LG infall onto Virgo (LEDA; Paturel et al. 1997); 1983I, see $\S 3.4 ;$ $1983 \mathrm{~N}$, Cepheids (Thim et al. 2003); 1998bw, z=0.0085 (Galama et al. 1998).

b 1982R, Clocchiatti et al. (1996); 1983I, 1983N, \& 1984L, Galactic Schlegel, Finkbeiner, \& Davis (1998); 1998bw, Patat et al. (2001).

${ }^{\mathrm{c}} 1982 \mathrm{R}, 26$ days before discovery (Porter \& Filippenko 1987); 1983I, see $\S 3.4 ; 1983 \mathrm{~N}, 2$ days before discovery and 16 days before B maximum (Porter \& Filippenko 1987); 1984L, 15 days before light maximum and by comparison with SN 1985F (Tsvetkov 1987; Schlegel \& Kirshner 1989); 1998bw, GRB 980425 (Galama et al. 1998). 